\title{
Parámetros genéticos de características de tipo y producción en ganado Holstein del departamento de Antioquia
}

\section{Genetic parameters of type traits and production in Holstein cattle from the Department of Antioquia}

\author{
Juan Corrales A, ${ }^{1,2}$ Zoot, Mario Cerón-Muñoz, ${ }^{1 *}$ Ph.D, Jhon Cañas A, ${ }^{1}$ Zoot, \\ Cristina Herrera $\mathrm{R}^{1}{ }^{1}$ Zoot, Samir Calvo $\mathrm{C}^{1}{ }^{1}$ Zoot.
}

\begin{abstract}
${ }^{1}$ Universidad de Antioquia, Facultad de Ciencias Agrarias. Grupo de Investigación en Genética, Mejoramiento y Modelación Animal, Medellin, Colombia. 2Joven investigador Colciencias, programa jóvenes investigadores e innovadores "Virginia Gutiérrez de Pineda", Medellin, Colombia.*Correspondencia: mceronm@agronica.udea.edu.co
\end{abstract}

Recibido: Mayo de 2010; Aceptado: Febrero de 2011.

\section{RESUMEN}

Objetivo. Estimar heredabilidades, correlaciones fenotípicas y genotípicas entre 24 características de tipo y producción de leche en vacas Holstein del departamento de Antioquia. Materiales y métodos. Se analizaron un total de 2395 registros de vacas Holstein. Se estimaron componentes de varianza mediante el procedimiento de máxima verosimilitud restricta con modelos animales bicaracterísticos. Para las características de tipo, el modelo incluyó los efectos fijos de finca, año de nacimiento y país de origen del padre de la vaca, el grupo contemporáneo (año-mes-clasificador) y la covariable edad a la clasificación. Para la producción de leche el modelo incluyó los efectos fijos de finca, año de parto, país y año de nacimiento del padre. Resultados. Las heredabilidades encontradas para las características de tipo variaron de 0.04 a 0.44 y para producción de leche fue de 0.22 , los errores estándar de la heredabilidad fueron inferiores a 0.06 . Las correlaciones genéticas de producción de leche con las características profundidad de la ubre $(-0.72)$ fue alta, mientras las correlaciones más altas entre las características de tipo fueron entre extremo anterior y tamaño (0.90), ángulo de la pezuña y profundidad del talón (0.98), profundidad del talón y uniformidad de pezuñas (0.85); y ligamento suspensorio medio y colocación de pezones posteriores (0.76). Conclusiónes. Se encontró una considerable variación genética en características de tipo dentro de la población Holstein de Antioquia. Las correlaciones genéticas entre algunas características de tipo fueron altas e indican la posibilidad de reducir el número de características evaluadas en cada animal.

Palabras clave: Correlaciones genéticas, ganado lechero, heredabilidad (Fuente:CAB, AIMS). 


\begin{abstract}
Objective. To estimate heritability, phenotypic and genetic correlations among 24 type traits and milk production in Holstein cows from Antioquia. Materials and methods. The data analyzed included 2395 holstein cow records. Variance components were estimated through the procedure of maximum restricted likelihood using bi-characteristic animal models. For the type traits, the model included the fixed effects such as, herd, father's year of birth and country of origin, contemporary group (year-month-classifier) and the classification age covariate. For milk production the model included fixed effects such as herd, calving year and father's year and country of birth. Results. The heritability found for the type traits varied from 0.04 to 0.44 and for milk production was 0.22 , standard errors for heritability were lower than 0.06 . Genetic correlations between milk production and udder depth $(-0.72)$ were high, while the highest correlations for the type traits were between height at front end and size (0.90), foot angle and heel depth (0.98), heel depth and hoof uniformity $(0.85)$ and median suspensory ligament with rear teat placement $(0.76)$. Conclusions. Considerable genetic variation in type traits in Holstein populations from Antioquia was found. Genetic correlations between some type traits were high and indicate the possibility of reducing the number of features evaluated in each animal.
\end{abstract}

Key words: Dairy cattle, genetic correlation, heritability (Source:CAB, AIMS).

\section{INTRODUCCIÓN}

Tradicionalmente la selección genética se ha realizado para producción de leche, hecho que ha provocado una reducción en el mérito genético de la salud y fertilidad en vacas lecheras $(1,2)$.

Las características de tipo (CT) se han propuesto como indicadores indirectos para el mejoramiento de los parámetros productivos, reproductivos y sanitarios (3-6). La clasificación lineal es el procedimiento por el cual se valora visualmente cada una de las CT de un individuo, donde cada característica se describe en un rango de 1 a 9 y se clasifican en grupos asociados con el cuerpo, anca, patas y pezuñas, y ubre (7).

La estimación de heredabilidad y correlaciones genéticas es importante para la realización de un programa de mejoramiento $(2,8)$. Las heredabilidades para las características del cuerpo se han encontrado en el rango de 0,10 a $0.43(7,9)$, para las características de patas y pezuñas un rango de 0.12 a $0.17(2,7)$ y las características de la ubre se encuentran en el rango de 0.13 a $0.38(2,7)$.

Diferentes estudios han mostrado la existencia de correlaciones genotípicas medias entre la producción de leche con estatura (0.42), profundidad del cuerpo (0.36), angularidad $(0.48)$, ancho de isquiones (0.46), altura de la ubre posterior $(0.48)$, e inserción anterior de la ubre (0.32) (7).

Internacionalmente se ha propuesto la utilización de índices de selección, los cuales incluyen la producción de leche y características de tipo (CT) que se relacionan con la producción, reproducción y salud de la vaca con el objetivo de lograr un progreso genético que conduzca a un mejoramiento de la productividad y la funcionalidad de las vacas lecheras en los hatos (10).

El objetivo de este estudio fue estimar heredabilidades, correlaciones genéticas y fenotípicas entre las características de tipo evaluadas en vacas de primer parto en el departamento de Antioquia.

\section{MATERIALES Y METODOS}

Sitio de estudio y caracterización de la muestra. Se utilizaron 2395 registros de vacas Holstein de primer parto, pertenecientes a 49 hatos ganaderos del departamento de Antioquia, con clasificación lineal realizada entre los años 2000 y 2008 por la Asociación Holstein de Colombia. Cada registro contenía la información de producción de leche ajustada a 305 días y edad adulta (11) y de 24 características de tipo las cuales fueron evaluadas asignándole un puntaje de 1 a 9 a cada característica (Tabla $1)$. Los padres de las vacas eran de diferentes orígenes: 44 de Canadá, 21 de Colombia y 212 de Estados Unidos

Estimación de los parámetros genéticos. Para las características de tipo se utilizó un modelo animal bi-característico que incluyó los efectos fijos de finca, año de nacimiento del padre, país de nacimiento del padre y grupo contemporáneo (año-ronda-clasificador), la covariable edad a la clasificación y el efecto aleatorio del animal. Para la producción de leche se consideró en el modelo los efectos 
Tabla 1. Abreviaturas y estadística descriptiva para las características de tipo y producción de leche en vacas Holstein del departamento de Antioquia.

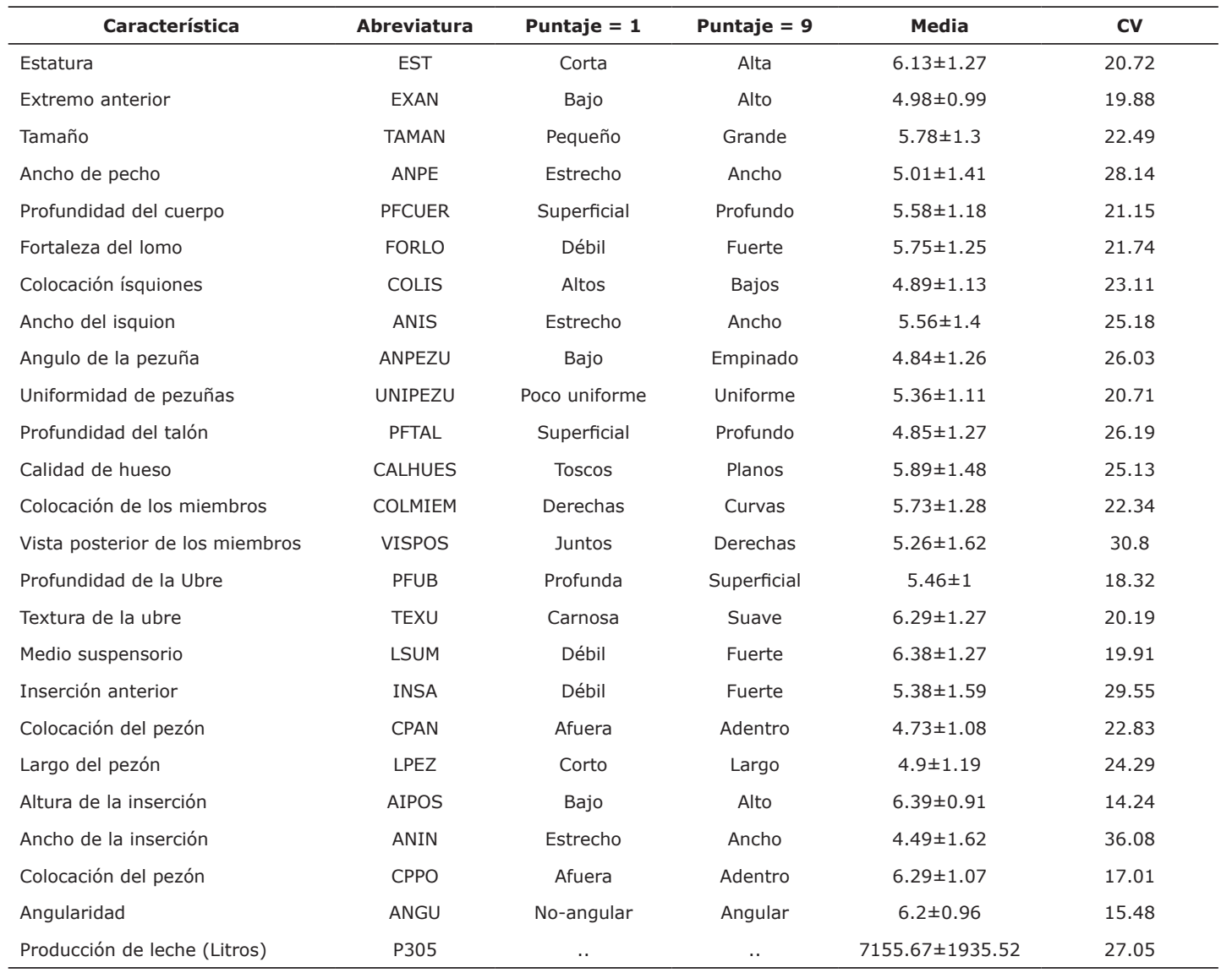

fijos finca, año de parto, año de nacimiento del padre y país de nacimiento del padre, y el efecto aleatorio del animal. El análisis fue realizado por el procedimiento máxima verosimilitud restricta libre de derivadas mediante el software MTDFREML desarrollado por Boldman et al (12).

$$
y_{i}=X \beta_{i}+Z \alpha_{i}+e_{i}
$$

Donde,

i=Característica de tipo desde la 1 hasta la característica de tipo 24 o producción de leche.

$X \beta_{i}=\beta_{i}$ son los efectos fijos de finca, año de nacimiento del padre, país de nacimiento de padre, grupo contemporáneo (año-rondaclasificador) y edad a la clasificación para las características de tipo, para producción de leche los efectos fijos de finca, año de parto, año de nacimiento del padre y país de nacimiento del padre.

Zai $=$ es el efecto aleatorio del animal

$\mathrm{e} i=$ Residuos.
Análisis de componentes de varianza. Se realizaron cuatro análisis de componentes de varianza separados en grupos que relacionaban la ubicación de las características de tipo en el animal. De esta manera, el cuerpo incluyó las características (estatura, extremo anterior, tamaño, ancho de pecho, profundidad del cuerpo y fortaleza del lomo), el anca incluyó (ancho de ísquiones y colocación de ísquiones), las patas y pezuñas (ángulo de la pezuña, uniformidad de pezuñas, profundidad del talón, calidad de hueso, colocación de los miembros y vista posterior de los miembros) y la ubre incluyó (profundidad de la ubre, textura de la ubre, ligamento suspensorio medio, inserción anterior, colocación de pezones anteriores, largo del pezón, altura de la inserción posterior, ancho de la inserción y colocación de pezones posteriores). Las heredabilidades se obtuvieron por medio de análisis uní-característicos.

Las correlaciones genéticas y fenotípicas entre las características de cada grupo se realizaron por medio de análisis bi-caracteristicos. En estos análisis se utilizaron para todas las características los mismos efectos fijos. 
Para estimar las correlaciones fenotípicas entres las diferentes características, se utilizó la siguiente ecuación de acuerdo con Falconer y Mackay (13).

$r p_{1,2}=\left(r g_{1,2} x \sqrt{h_{1}^{2}} x \sqrt{h_{2}^{2}}\right)+\left(r a_{1,2} x \sqrt{1-h_{1}^{2}} x \sqrt{1-h_{2}^{2}}\right)$

Donde:

$\mathrm{rp}_{1,2}=$ Correlación fenotípica entre las características 1 y 2

$\mathrm{rg}_{1,2}=$ Correlación genética entre las características 1 y 2

$\mathrm{ra}_{1,2}=$ Correlación ambiental entre las características 1 y 2

$h_{1}^{2}=$ heredabilidad de la característica 1

$h_{2}^{2}=$ heredabilidad de la característica 2

\section{RESULTADOS}

Las medias y desviaciones estándar de las características de tipo (CT) se pueden observar en la tabla 1. La media para la producción de leche fue de $7155.67 \pm 1935.52$. La característica ancho de la inserción presentó la mayor variabilidad (36.08), el menor coeficiente de variación fue en la característica altura de la inserción posterior (14.24)

En la tabla 2 se puede observar las heredabilidades, correlaciones genéticas y fenotípicas para las características del cuerpo. Las heredabilidades variaron entre 0.17 para ancho de pecho y 0.37 para estatura, la correlación genética varió de -0.30 entre producción de leche y extremo anterior a 0.90 entre tamaño y extremo anterior, mientras que las correlaciones fenotípicas se encontraron entre 0.03 a 0.58 .
Las características colocación de ísquiones y ancho de ísquiones tuvieron la misma heredabilidad $(0.44)$ con un error estándar de 0.062 y 0.058 , respectivamente. La correlación genética de la característica colocación de ísquiones con P305 fue de 0.02, mientras el ancho de ísquiones tuvo una correlación genética de 0.04 con P305 (Tabla 3).

Tabla 3. Heredabilidades de las características del anca (sobre la diagonal), correlaciones fenotípicas (en la diagonal) y correlaciones genéticas (encima de la diagonal) evaluadas en vacas Holstein de Antioquia.

\begin{tabular}{lccc}
\hline & \multicolumn{3}{c}{ Características } \\
\cline { 2 - 4 } & COLIS & ANIS & P305 \\
\hline COLIS & $0.44 \pm 0.06$ & $0.23 \pm 0.11$ & $0.15 \pm 0.04$ \\
ANIS & 0.07 & $0.44 \pm 0.06$ & $0.09 \pm 0.15$ \\
P305 & 0.02 & 0.04 & $0.17 \pm 0.00$ \\
\hline COLIS=Colocación de ísquiones, ANIS=Ancho & de ísquiones,
\end{tabular}

P305=Producción de leche.

Las heredabilidades para patas y pezuñas variaron entre 0.04 para vista posterior y 0.22 para calidad de hueso. Se presentó una alta correlación genética entre profundidad del talón con ángulo de la pezuña (0.98) y uniformidad de pezuñas (0.85) y una correlación negativa entre colocación de los miembros con profundidad del talón y vista posterior de los miembros $(-0.46$ y -0.45 , respectivamente) como se puede observar en la tabla 4.

Las heredabilidades para las características de ubre (Tabla 5) variaron de 0.12 a 0.28 las cuales fueron de medias a bajas. El ligamento suspensorio medio presentó una

Tabla 2. Heredabilidades de las características del cuerpo (en la diagonal), correlaciones fenotípicas (debajo de la diagonal) y correlaciones genéticas (encima de la diagonal) evaluadas en vacas Holstein de Antioquia.

\begin{tabular}{lccccccccc}
\hline & \multicolumn{7}{c}{ Características } \\
\cline { 2 - 8 } & EST & EXAN & TAMAN & ANPE & PFCUER & FORLO & ANGU & PL305EM \\
\hline EST & $0.37 \pm 0.06$ & $0.39 \pm 0.15$ & $0.69 \pm 0.10$ & $0.01 \pm 0.18$ & $0.47 \pm 0.15$ & $0.69 \pm 0.10$ & $0.50 \pm 0.13$ & $0.02 \pm 0.17$ \\
EXAN & 0.22 & $0.22 \pm 0.05$ & $0.90 \pm 0.11$ & $0.42 \pm 0.18$ & $0.63 \pm 0.18$ & $-0.19 \pm 0.23$ & $0.24 \pm 0.18$ & $-0.30 \pm 0.22$ \\
TAMAN & 0.54 & 0.38 & $0.20 \pm 0.05$ & $0.58 \pm 0.14$ & $0.74 \pm 0.13$ & $-0.01 \pm 0.24$ & $0.52 \pm 0.17$ & $-0.09 \pm 0.22$ \\
ANPE & 0.14 & 0.24 & 0.58 & $0.17 \pm 0.05$ & $0.61 \pm 0.16$ & $-0.17 \pm 0.25$ & $0.03 \pm 0.19$ & $0.18 \pm 0.22$ \\
PFCUER & 0.29 & 0.21 & 0.52 & 0.39 & $0.18 \pm 0.05$ & $0.05 \pm 0.25$ & $0.70 \pm 0.14$ & $-0.03 \pm 0.07$ \\
FORLO & 0.54 & 0.05 & 0.08 & 0.03 & 0.17 & $0.20 \pm 0.05$ & $0.26 \pm 0.21$ & $-0.16 \pm 0.27$ \\
ANGU & 0.35 & 0.2 & 0.23 & 0.08 & 0.34 & 0.28 & $0.24 \pm 0.06$ & $0.14 \pm 0.19$ \\
PL305EM & 0.08 & 0.04 & 0.13 & 0.12 & 0.18 & 0.07 & 0.19 & $0.17 \pm 0.00$ \\
\hline
\end{tabular}

$\mathrm{EST}=$ Estatura, $\mathrm{EXAN}=$ Extremo anterior, TAMAN $=$ Tamaño, ANPE = Ancho de pecho, PFCUER=Profundidad del cuerpo, FORLO=Fortaleza del lomo, ANGU=Angularidad, PL305EM=Producción de leche ajustada a 305 días y edad madura. 
Tabla 4. Heredabilidades de las características de patas y pezuñas (en la diagonal), correlaciones fenotípicas (debajo de la diagonal) y correlaciones genéticas (encima de la diagonal) evaluadas en vacas Holstein de Antioquia.

\begin{tabular}{lcccccccc}
\hline & \multicolumn{7}{c}{ Características } \\
\cline { 2 - 7 } & ANPEZU & UNIPEZU & PFTAL & CALHUES & COLMIEN & VISPOS \\
\hline ANPEZU & $0.14 \pm 0.05$ & $0.46 \pm 0.34$ & $0.98 \pm 0.09$ & $-0.46 \pm 0.23$ & $-0.23 \pm 0.23$ & $0.41 \pm 0.37$ & $0.07 \pm 0.23$ \\
UNIPEZU & 0.19 & $0.05 \pm 0.04$ & $0.85 \pm 0.41$ & $-0.12 \pm 0.31$ & $-0.04 \pm 0.33$ & $0.67 \pm 0.56$ & $0.13 \pm 0.33$ \\
PFTAL & 0.59 & 0.20 & $0.12 \pm 0.04$ & $-0.20 \pm 0.23$ & $-0.46 \pm 0.21$ & $0.56 \pm 0.38$ & $0.19 \pm 0.24$ \\
CALHUES & -0.1 & -0.01 & -0.09 & $0.22 \pm 0.06$ & $0.15 \pm 0.20$ & $-0.02 \pm 0,34$ & $-0.13 \pm 0.21$ \\
COLMIEN & -0.05 & -0.01 & -0.22 & 0.11 & $0,17 \pm 0.05$ & $-0.45 \pm 0.31$ & $0.01 \pm 0.21$ \\
VISPOS & 0.09 & -0.01 & 0.12 & -0.01 & -0.26 & $0.04 \pm 0.04$ & $0.35 \pm 0.35$ \\
P305 & 0.04 & 0.04 & 0.09 & 0.01 & -0.04 & 0.10 & $0.17 \pm 0.00$ \\
\hline
\end{tabular}

ANPEZU=Angulo de la pezuña; UNIPEZU= Uniformidad de pezuñas; PFTAL=Profundidad del talón; CALHUES=Calidad de hueso; COLMIEM=Colocación de los miembros; VISPOS=Vista posterior de los miembros.

Tabla 5. Heredabilidades de las características de la ubre (en la diagonal), correlaciones fenotípicas (debajo de la diagonal) y correlaciones genéticas (encima de la diagonal) evaluadas en vacas Holstein de Antioquia.

\begin{tabular}{|c|c|c|c|c|c|c|c|c|c|c|}
\hline & \multicolumn{10}{|c|}{ Características } \\
\hline & PFUB & TEXU & LSUM & INSA & CPAN & LPEZ & AIPOS & ANIN & CPPO & P305 \\
\hline PFUB & $\begin{array}{c}0.18 \pm \\
0.05\end{array}$ & $\begin{array}{c}0.50 \\
\pm 0.21\end{array}$ & $\begin{array}{c}0.25 \\
\pm 0.20\end{array}$ & $\begin{array}{c}0.55 \\
\pm 0.17\end{array}$ & $\begin{array}{c}0.29 \\
\pm 0.17\end{array}$ & $\begin{array}{l}-0.29 \\
\pm 0.16\end{array}$ & $\begin{array}{c}0.25 \\
\pm 0.22\end{array}$ & $\begin{array}{l}-0.27 \\
\pm 0.19\end{array}$ & $\begin{array}{c}0.58 \\
\pm 0.17\end{array}$ & $\begin{array}{l}-0.72 \\
\pm 0.13\end{array}$ \\
\hline TEXU & 0.09 & $\begin{array}{c}0.15 \\
\pm 0.05\end{array}$ & $\begin{array}{c}0.51 \\
\pm 0.18\end{array}$ & $\begin{array}{c}0.38 \\
\pm 0.19\end{array}$ & $\begin{array}{c}0.40 \\
\pm 0.16\end{array}$ & $\begin{array}{l}-0.16 \\
\pm 0.18\end{array}$ & $\begin{array}{c}0.52 \\
\pm 0.18\end{array}$ & $\begin{array}{c}0.22 \\
\pm 0.20\end{array}$ & $\begin{array}{c}0.38 \\
\pm 0.18\end{array}$ & $\begin{array}{c}0.03 \\
\pm 0.23\end{array}$ \\
\hline LSUM & 0.05 & 0.30 & $\begin{array}{c}0.17 \\
\pm 0.05\end{array}$ & $\begin{array}{c}0.24 \\
\pm 0.20\end{array}$ & $\begin{array}{c}0.26 \\
\pm 0.17\end{array}$ & $\begin{array}{l}-0.10 \\
\pm 0.18\end{array}$ & $\begin{array}{c}0.57 \\
\pm 0.23\end{array}$ & $\begin{array}{c}0.36 \\
\pm 0.20\end{array}$ & $\begin{array}{c}0.76 \\
\pm 0.17\end{array}$ & $\begin{array}{c}0.38 \\
\pm 0.43\end{array}$ \\
\hline INSA & 0.17 & 0.29 & 0.10 & $\begin{array}{c}0.17 \\
\pm 0.45\end{array}$ & $\begin{array}{c}0.08 \\
\pm 0.18\end{array}$ & $\begin{array}{l}-0.04 \\
\pm 0.18\end{array}$ & $\begin{array}{c}0.57 \\
\pm 0.21\end{array}$ & $\begin{array}{c}0.05 \\
\pm 0.20\end{array}$ & $\begin{array}{c}0.02 \\
\pm 0.19\end{array}$ & $\begin{array}{l}-0.27 \\
\pm 0.19\end{array}$ \\
\hline CPAN & 0.05 & 0.14 & 0.23 & 0.18 & $\begin{array}{c}0.25 \\
\pm 0.05\end{array}$ & $\begin{array}{l}-0.05 \\
\pm 0.15\end{array}$ & $\begin{array}{c}0.36 \\
\pm 0.20\end{array}$ & $\begin{array}{l}-0.03 \\
\pm 0.18\end{array}$ & $\begin{array}{c}0.42 \\
\pm 0.15\end{array}$ & $\begin{array}{l}-0.15 \\
\pm 0.05\end{array}$ \\
\hline LPEZ & -0.07 & -0.01 & -0.04 & -0.01 & -0.07 & $\begin{array}{c}0.28 \\
\pm 0.05\end{array}$ & $\begin{array}{c}0.15 \\
\pm 0.19\end{array}$ & $\begin{array}{c}0.05 \\
\pm 0.18\end{array}$ & $\begin{array}{l}-0.37 \\
\pm 0.15\end{array}$ & $\begin{array}{c}0.16 \\
\pm 0.04\end{array}$ \\
\hline AIPOS & 0.16 & 0.24 & 0.12 & 0.13 & $-0,02$ & 0.01 & $\begin{array}{c}0.12 \\
\pm 0.04\end{array}$ & $\begin{array}{c}0.34 \\
\pm 0.21\end{array}$ & $\begin{array}{c}0.40 \\
\pm 0.21\end{array}$ & $\begin{array}{l}-0.19 \\
\pm 0.21\end{array}$ \\
\hline ANIN & -0.12 & 0.14 & 0.09 & 0.05 & 0.04 & -0.01 & 0.20 & $\begin{array}{c}0.16 \\
\pm 0.04\end{array}$ & $\begin{array}{l}-0.04 \\
\pm 0.19\end{array}$ & $\begin{array}{c}0.32 \\
\pm 0.19\end{array}$ \\
\hline СРPO & 0.02 & 0.16 & 0.36 & 0.06 & 0.31 & -0.1 & 0.02 & 0.02 & $\begin{array}{c}0.22 \\
\pm 0.05\end{array}$ & $\begin{array}{l}-0.34 \\
\pm 0.19\end{array}$ \\
\hline P305 & -0.30 & 0.07 & 0.08 & -0.02 & 0.02 & 0.08 & 0.05 & 0.04 & 0.06 & $\begin{array}{c}0.17 \\
\pm 0.00\end{array}$ \\
\hline
\end{tabular}

PFUB=Profundidad de la ubre; TEXU=Textura de la ubre; LSUM=Ligamento suspensorio medio; INSA=Inserción anterior; CPAN=Colocación de pezones anteriores; LPEZ=Largo de pezones; AIPOS=Altura de la inserción posterior; ANIN=Ancho de la inserción; CPPO=Colocación de pezones posteriores.

alta correlación con colocación de los pezones posteriores $(0.76)$ y altura de la inserción posterior (0.57), igualmente se presentó una correlación alta entre profundidad de la ubre con colocación de pezones posteriores $(0.58)$ y textura de la ubre (0.50). La característica ancho de la inserción presentó una correlación positiva $(0.34)$ con producción de leche.

\section{DISCUSIÓN}

En este estudio las heredabilidades de las características del cuerpo se encontraron entre 0.17 (ancho de pecho) y 0.37 (estatura). La heredabilidad para ancho de pecho difiere de otros estudios en los cuales la heredabilidad encontrada estuvo entre 0.26 y 0.36 , mientras para la estatura se encontró un valor similar al reportado por otros investigadores con un rango entre 0.35 y $0.59(2,9,14)$. La heredabilidad para la profundidad del cuerpo $(0.18)$ no difiere de manera significativa de la encontrada en otros estudios con un rango de 0.20 a 0.34 $(2,6,9,14)$.

Las correlaciones genéticas entre tamaño y estatura (0.69), y tamaño y profundidad del cuerpo (0.74) se encuentran cercanas a las reportadas por Degroot et al (5). La angularidad o forma lechera y profundidad del cuerpo, cuya correlación genética encontrada en este estudio fue de 0.70 es similar a lo reportado por Berry et al (7) y diferente a la reportada por DeGroo et al (5) quienes encontraron una correlación genética de -0.07 . Estas diferencias pueden explicarse debido al tipo de selección que por años se ha llevado a cabo en Colombia debido 
a que el productor ha seleccionado estas dos características de una manera conjunta y por ende las correlaciones genéticas son altas y positivas. Las características ancho de pecho y angularidad presentaron correlaciones positivas con producción de leche, las cuales están de acuerdo con estudios previos $(5,7,15)$.

Las características colocación de isquiones y ancho de isquiones no presentaron una alta correlación genética con producción de leche. Wall et al (16) encontraron que vacas con colocación de los isquiones altos presentan mayor intervalo entre partos y vacas anchas de isquiones presentan una mayor actividad luteal. Lo anterior indica que en Antioquia se puede realizar selección para la característica colocación y ancho de Ísquiones favoreciendo la eficiencia reproductiva sin afectar la producción de leche.

Para las características de patas y pezuñas importantes en los sistemas de pastoreo debido a que las vacas necesitan una mejor locomoción que permita un pastoreo más eficiente y movilidad del potrero al sitio de ordeño (7) se encontraron heredabilidades para el ángulo de la pezuña y colocación de los miembros de 0.14 y 0.17 , respectivamente; los cuales coinciden con resultados reportados para la raza Holstein $(2,3,6)$.

La alta correlación genética entre profundidad del talón y ángulo de la pezuña (0.98) y profundidad del talón y uniformidad de las pezuñas $(0.85)$ está indicando la alta relación genética que existe entre las características y que el aumento en la profundidad del talón genéticamente aumenta el ángulo y la uniformidad de pezuñas.

La correlación de las características de las patas y pezuñas con producción de leche se encontró en un rango de -0.13 con calidad de hueso y 0.35 con vista posterior de los miembros. Algunas de las correlaciones estimadas en este estudio concuerdan con las reportadas por Berry et al (7) quienes encontraron correlaciones genéticas de 0.07 entre ángulo de la pezuña y producción de leche. La correlación genética encontrada entre vista posterior de los miembros y producción de leche indica la relación que existe debido a que vacas con baja calificación pueden tener menores producciones de leche debido a la movilidad reducida que presentan (17).

Las heredabilidades para las características de la ubre estuvieron en el rango de 0.12 a 0.28 .
La heredabilidad para todas las características son cercanas a las encontradas por Dal Zotto et al (2) quienes reportaron heredabilidades en un rango de 0.12 a 0.24 . La característica ligamento suspensorio medio presentó una heredabilidad de 0.52 en el estudio realizado por DeGroot et al (5), mientras en el de Berry et al (7) la heredabilidad fue inferior a 0.18 al igual que el encontrado en este estudio donde la heredabilidad para ligamento suspensorio fue de 0.17 .

Las características profundidad de la ubre, ligamento suspensorio medio, inserción anterior, ancho de la inserción y colocación de pezones posteriores fueron las que presentaron mayor correlación genética con producción de leche. Las correlaciones negativas indican que vacas con alta producción tienen una ubre más débil debido a que presenta mayor profundidad, una inserción anterior débil y pezones posteriores hacia afuera.

Teniendo en cuenta que en la clasificación lineal una calificación de 9 para profundidad de la ubre corresponde a una ubre superficial y la calificación de 1 corresponde a una ubre profunda. La correlación genética negativa entre profundidad de la ubre y producción de leche, indica que vacas con ubres muy profundas pueden tener mayor producción pero presentar mayores problemas sanitarios en la ubre y por ende un mayor riesgo de descarte como lo indicaron Nash et al (18).

La correlación positiva entre las características ancho de la inserción y ligamento suspensorio medio se presentan porque ubres anchas de la inserción se relacionan con mayor capacidad de almacenamiento de la leche. Además, el productor ha seleccionado vacas de alta producción con ligamentos suspensorios fuertes que garanticen una mayor permanencia del animal en el hato (6).

Los resultados de este estudio indicaron una considerable variación genética existente para las características de tipo dentro de la población Holstein de Antioquia. Las correlaciones genéticas encontradas entre algunas características de tipo indican la posibilidad de reducir el número de características a evaluar en cada animal. Características como ángulo de la pezuña y profundidad del talón con una correlación genética de 0.98 están indicando que la selección para una de las dos características estaría involucrando la selección para la otra característica.

Las correlaciones genéticas encontradas para 
producción de leche y las características de tipo indican que la conformación del animal tienen de media a baja relación genética con producción de leche, a excepción de la característica profundidad de la ubre que tuvo una alta correlación negativa que indica que las vacas de alta producción tienden a tener unas ubres más profundas por efecto del peso de la leche.

Lo anterior indica que es posible seleccionar individuos para características de tipo sin afectar la producción de leche y de esta manera tener vacas funcionales con una larga vida productiva y pocos problemas sanitarios.

\section{Agradecimientos}

Este trabajo fue posible gracias al apoyo del Ministerio de Agricultura y Desarrollo Rural, FEDEGAN. Universidad de Antioquia, Asociación Holstein de Colombia y Corporación Antioquia Holstein.

\section{REFERENCIAS}

1. Castillo-Juarez H, Oltenacu PA, Blake RW, McCulloch, Cienfuego-Rivas. Effect of herd environment on genetic and phenotypic relationships among milk yield, conception rate and somatic cell score in Holstein cattle. J Dairy Sci 2000; 83:807-814.

2. Dal Zotto $R$, Marchi $M$, Darvit C, Cassandro M, Gallo L, Carnier P, Bittante G. Heritabilities and genetic correlation of body condition score and calving interval with yield, somatic cell score, and linear type traits in Brown Swiss cattle. J Dairy Sci 2007; 90:5737-5743.

3. Perez-Cabal MA, Garcia C, Gonzáles-Recio O, Alenda R. Genetic and phenotypic relationships among locomotion type trait, profit, reproduction, longevity and fertility in Spanish dairy cows. J Dairy Sci 2006; 89:1776-1783.

4. Wang Y, Stella A, Boettcher PJ. Genetic analysis of detective type characteristics and their genetic relationships with herd life of Canadian Holstein. J Dairy Sci 2002; 85.

5. DeGroot BJ, Keown JF, Van Vleck LD, Marotz EL. Genetic parameters and somatic cell scores to divergent selection for predicted transmitting ability for type in Holstein. J Dairy Sci 2002; 85:1578-1585.

6. Pérez-Cabal MA, Alenda R. Genetic relationships between lifetime profit and type traits in Spanish Holstein. J Dairy Sci 2002; 85:3480-3491.
7. Berry DP, Buckley F, Dillon P, Evans RD, Veerkamp RF. Genetic relationships among linear type traits, milk yield, body weight, fertility and somatic cell count in primiparous dairy cows. Ir J Agr Foot Res 2004; 43:161-176.

8. Valencia M, Ruiz $F$, Montaldo HH. Estimación de parámetros genéticos para características de longevidad y producción de leche en ganado Holstein en México. Interciencia $2004 ; 29: 52-56$

9. Dechow $C D$, Rogers GW, Klei L, Lawlor TJ. Heritabilities and correlations among body condition score, dairy form and selected linear type trait. J Dairy Sci 2003; $86: 2236-2242$.

10. Interbull guidelines for national \& international genetic evaluation systems in dairy cattle with focus on production traits. National evaluation systems information. Interbull [en línea] 2001 Noviembre [fecha de acceso 09 de Junio de 2010]; 28. URL disponible en: http://www-interbull.slu. se/bulletins/framesida-pub.htm

11. Stanton TL. Investigation of genotype by environment interaction for Holstein milk yield in Colombia, Mexico and Puerto Rico. [Tesis de Doctorado]. Ithaca, Estados Unidos: Cornell University, Department of Animal Science: 1990.

12. Boldman KG, Kriese LA, Van Vleck D, Van Tassell CP, Kachman SD. A manual for use of MTDFREML. A set of programs to obtain estimates of variances and covariances (Draft). USDA, Agric Res Serv 1995:114. 
13. Falconer DS, Mackay TF. Introducción a la genética cuantitativa, Zaragoza, España: Acribia; 2001.

14. Valencia M, Montaldo HO, Ruiz F. Parámetros genéticos para características de conformación, habilidad de permanencia y producción de leche en ganado Holstein en mexico. Téc Pecu Méx 2008; 46(3):235-248.

15. Brotherstone S. Genetic and phenotypic correlations between linear type traits and production traits in Holstein-Friesian dairy cattle. Anim Prod 1994; 59:183-187.

16. Wall E, White MS, Coffey MP, Brotherstone $S$. The relationship between fertility, rump angle, and Selected type information in Holstein-Friesian cows. J Dairy Sci 2005; 88:1521-1528.
17. Boettcher PJ, Jairath LK, Dekkers JCM. Alternative methods for genetic evaluation of sires for survival of their daughters in the first three lactations. 6th World Congr. Genet. Appl Livest Prod 1998; 23:363-366.

18. Nash DL, Rogers GW, Cooper JB, Hargrove GL, Keown JF. Relationships among severity and duration of clinical mastitis and sire transmitting abilities for somatic cell score, udder type trait, productive life, and protein yield. J Dairy Sci 2002; 85:1273-1284. 